\title{
A COVID-19 e o Princípio da Responsabilidade Social e Saúde: uma análise crítica
}

\author{
COVIID-19 and the Principle of Social Responsibility and Health: a critical analysis \\ COVID-19 y el Principio de Responsabilidad Social y Salud: un análisis crítico
}

Recebido: 14/10/2021 | Revisado: 23/10/2021 | Aceito: 01/11/2021 | Publicado: 02/11/2021

\author{
Maria Luiza Rêgo Bezerra \\ ORCID: https://orcid.org/0000-0002-3336-7760 \\ Universidade de Brasília, Brasil \\ E-mail: marialuizaregobezerra@gmail.com \\ Helena Eri Shimizu \\ ORCID: https://orcid.org/0000-0001-5612-5695 \\ Universidade de Brasília, Brasil \\ E-mail: shimizu@unb.br
}

\begin{abstract}
Resumo
Constitucionalmente a saúde é direito de todos e dever do Estado no Brasil o que ressalta a Responsabilidade Social do mesmo em prover saúde para a população brasileira, sem qualquer juízo de valor ou discriminação. Entretanto, observa-se uma realidade diferente do que está preconizado normativamente, uma vez que, as iniquidades sociais, políticas e econômicas são sobressalentes à direitos fundamentais como o direito à saúde. A Declaração Universal sobre Bioética e Direitos Humanos dispõe em seu artigo 14 o princípio Responsabilidade Social e saúde como primordial para o exercício das ações do Estado em promover o exercício do direito à saúde à população brasileira, sobretudo, em situações emergenciais como a pandemia de COVID-19. Por isso objetivou-se analisar criticamente as ações estratégicas do governo brasileiro à luz do princípio Responsabilidade Social e saúde diante da pandemia de COVID-19. Observou-se a proximidade do Estado com a inviolabilidade do princípio Responsabilidade Social e saúde no contexto da pandemia da COVID-19 a partir de ações, políticas e determinações governamentais instituídas pelo próprio governo brasileiro.
\end{abstract}

Palavras-chave: Governo; Brasil; Responsabilidade social; Direito à saúde; Pandemias; Coronavírus.

\begin{abstract}
Constitutionally, health is everyone's right and the State's duty in Brazil, which highlights its social responsibility to provide health care for the Brazilian population, without any value judgment or discrimination. However, there is a different reality from what is normatively recommended, since social, political and economic inequalities are essential to fundamental rights such as the right to health. Article 14 of the Universal Declaration on Bioethics and Human Rights establishes the principle of Social responsibility and health as essential for the exercise of State actions to promote the exercise of the right to health for the Brazilian population, especially in emergency situations such as the pandemic of COVID. -19. Therefore, the objective was to critically analyze the strategic actions of the Brazilian government in light of the principle of social responsibility and health in view of the COVID-19 pandemic. The proximity of the State with the inviolability of the principle of Social Responsibility and health was observed in the context of the COVID-19 pandemic, based on government actions, policies and determinations instituted by the Brazilian government itself.
\end{abstract}

Keywords: Government; Brazil; Social responsability; Right to health; Pandemics; Coronavirus.

\section{Resumen}

Constitucionalmente, la salud es un derecho de todos y un deber del Estado en Brasil, lo que destaca su responsabilidad social de brindar atención médica a la población brasileña, sin ningún juicio de valor ni discriminación. Sin embargo, existe una realidad diferente a la recomendada normativamente, ya que las desigualdades sociales, políticas y económicas son fundamentales para derechos fundamentales como el derecho a la salud. El artículo 14 de la Declaración Universal sobre Bioética y Derechos Humanos establece el principio de responsabilidad social y salud como esencial para el ejercicio de las acciones del Estado para promover el ejercicio del derecho a la salud de la población brasileña, especialmente en situaciones de emergencia como la pandemia de COVID -19. Por lo tanto, el objetivo fue analizar críticamente las acciones estratégicas del gobierno brasileño a la luz del principio de responsabilidad social y salud ante la pandemia de COVID-19. La cercanía del Estado con la inviolabilidad del principio de Responsabilidad Social y salud se observó en el contexto de la pandemia del COVID19, con base en acciones, políticas y determinaciones gubernamentales instituidas por el propio gobierno brasileño. Palabras-clave: Gobierno; Brasil; Responsabilidade social; Derecho a la salud; Pandemias; Coronavirus. 


\section{Introdução}

A saúde como direito fundamental de todos e dever do estado é descrita na Constituição Federal de 1988 como primária e essencial a partir de políticas sociais e econômicas que visem a minimização de riscos de doenças e outros agravos além do acesso às ações de prevenção, promoção, recuperação e reabilitação da saúde para todos. Embora ainda precursora à Lei orgânica do SUS, a constituinte introduziu a importância da integralidade, universalidade e equidade do acesso à saúde e preponderância da ação do Estado para tanto (Brasil, 1988; 1990).

Nesta interlocução, tem-se o artigo 14 da Declaração Universal sobre Bioética e Direitos Humanos que apresenta o princípio Responsabilidade Social e Saúde como norma que consagra a competência do Estado em promover saúde e desenvolvimento social para toda população em conjuntura aos demais setores da sociedade e que, hodiernamente, no contexto da pandemia do novo coronavírus (Sars-CoV-2) decretada em 11 de março de 2020 pela Organização Mundial de Saúde (OMS), tem sido evidente as nuances de sua (in)violabilidade, uma vez que, a população brasileira enfrenta os desafios de acesso à saúde, sobretudo, mimetizados por medidas governamentais instáveis, negacionistas e insuficientes para a impactante problemática então ocasionada (UNESCO, 2005; WHO, 2020; Schaefer et al., 2020).

Sob o prisma da Bioética, recorda-se o modelo principialista com sobressalência do princípio da justiça quando se trata da promoção do direito à saúde e da responsabilização do Estado neste âmbito, também sendo relacionados os princípios de não-maleficência, beneficência e autonomia que deveria serem cerceadores das decisões governamentais tendo em vista a manutenção da dignidade humana com vistas à necessidade de cada um, ressaltando aos indivíduos em situação de maior vulnerabilidade, proposta está introduzida pela Bioética de Intervenção (BI), nativamente latinomaericana, em sua estrutura consequencialista, utilitarista e analítica da dimensão social como primordial para os processos saúde-doença e possíveis desequilíbrios neste (Beauchamp \& Childress, 1979; Feitosa \& Nascimento, 2015; Garrafa \& Porto, 2003) .

Com isto este trabalho objetiva analisar criticamente as ações estratégicas do governo brasileiro à luz do princípio Responsabilidade Social e saúde diante da pandemia de COVID-19, tendo como premissa a competência do Estado para o provimento de acesso à saúde de sua população, sobretudo, em situações graves e emergenciais como a pandemia do novo coronavírus (Sars-CoV-2) e, por conseguinte analisar sob o prisma da Bioética as relações ora evidenciadas ao princípio 14.

\section{Metodologia}

Trata-se de uma análise crítica das ações estratégias do governo brasileiro diante da pandemia de COVID-19, à luz do princípio Responsabilidade Social e saúde presente no artigo 14 da Declaração Universal sobre Bioética e Direitos Humanos (Unesco, 2005). Porquanto, a fim de facilitar o destrinchar da análise crítica, emergiram duas categorias temáticas a saber: Responsabilidade Social e saúde; O princípio, o Estado, a pandemia e a Bioética. A análise crítica trata-se de uma abordagem teórico-metodológica qualitativa que possibilita o melhor entendimento sobre algo ou algum evento (no caso desta pesquisa as ações governamentais no contexto da pandemia da COVID-19 a partir do princípio 14 da DUBDH) a afim de reforçar seus padrões epistemológicos (Borde, Hernández-Álvarez, Porto, 2015).

\section{Resultados e Discussão}

\subsection{Responsabilidade Social e saúde}

A temática da Responsabilidade Social é constantemente discutida nas bancadas das ciências humanas, sendo estas basilares para discussões que se projetam à outras ciências como visivelmente se observam nas ciências biomédicas e da saúde. É um processo transdisciplinar que possibilita a melhor compreensão e aplicabilidade de constructos essenciais para análise do 
dinamismo dos indivíduos, sobretudo, no contexto social. A justiça e a igualdade são premissas da Responsabilidade Social que também alcançam temáticas como meio ambiente e sustentabilidade (Silva \& Gomes, 2020).

Epistemologicamente, a Responsabilidade Social pode ser conceituada pelo conjunto de ações praticadas por uma entidade ou por pessoas, direcionadas para o bem-estar social embasadas por três eixos de sustentabilidade: social, cultural e ambiental com a pretensão de favorecer o desenvolvimento individual e coletivo o que traz à tona a Declaração Universal sobre Bioética e Seres Humanos que dispõe sobre a Responsabilidade Social e saúde como um dos princípios deste documento e introduz que "a promoção da saúde e do desenvolvimento social para a sua população é objetivo central dos governos, partilhado por todos os setores da sociedade" ratificando a responsabilidade estatal de promulgar ações equânimes de Responsabilidade Social ao setor saúde observando ao máximo os determinantes sociais de saúde, envolvidos neste processo e favorecendo a atuação compartilhada com os usuários de participarem no desenvolvimento e aplicação das ações voltadas para o acesso à saúde (UNESCO, 2005; Silva \& Gomes, 2020; Carrapato, Correia, Garcia, 2017).

Todos os cidadãos capazes devem ter Responsabilidade Social, uma vez que, uma atitude ou omissão individual poderá trazer consequências ao coletivo, minimizando o respeito aos demais direitos humanos além do direito à saúde com consequentes disparidades que poderão se tornar ainda mais nocivas, sobretudo, aos indivíduos que são ou estão em situação de vulnerabilidade.

Os princípios doutrinários e organizacionais do Sistema Único de Saúde (SUS) reforçam a Responsabilidade Social quando ensejam a universalidade, integralidade e equidade como primordiais para o acesso à saúde, bem como a hierarquização, participação popular, territorialização, descentralização e comando único para a fundamentação das políticas que devem ser normatizadas para o máximo de acesso de pessoas, famílias e comunidades ao SUS (Brasil, 1990). A perspectiva é que todos os princípios estejam interrelacionados pela ação do Estado o que pode configurar o exercício de sua Responsabilidade Social. O acesso universal é um dos maiores desafios, pois questiona-se a real aplicabilidade deste princípio tendo em vista a diversidade cultural e de território brasileiro e a gestão, muitas vezes empecilho para o norteamento de ações que corroborem com este princípio. A integralidade traz à tona que todo e qualquer cidadão deve ter acesso à saúde em todos os níveis de assistência (primário, secundário e terciário) conforme as suas necessidades, casando assim com o princípio doutrinário da equidade que visa tratar com desigualdade os desiguais, corroborando assim com a proposta da corrente de pensamento periférica, latino-americana, Bioética da Intervenção (BI) que será abordada posteriormente (Mattos, 2009; Duarte, Eble, Garcia, 2018; Paim, 2019).

Pensando-se em hierarquizar os princípios o ideal seria a equidade como basilar para que a integralidade e, então a universalidade fossem as mais concretas possíveis, pois são justamente as questões de exclusão voltadas para a maioria da população brasileira, obstáculos para o exercício do direito à saúde. Da parte do Estado cabe ressaltar a importância de se incentivar e discutir os princípios do SUS, no real conceito de cada um pensando na real concretude destes de responsabilização das três esferas de governo: municipal, estadual e federal.

O prisma biopsicossocial que conceitua a saúde não somente como ausência de doenças corrobora com a importância da dizimação dos princípios doutrinários do SUS, desde o controle social, passando pelos profissionais de saúde até a gestão, que também são representantes, além de outros setores da sociedade, do cumprimento da reponsabilidade social e saúde. Todas as políticas públicas de saúde em seus conteúdos referem-se à responsabilidade de cada uma das três esferas de governo diante daquela problemática de saúde, desde a atenção à saúde da criança e do adolescente, perpassando pela saúde do homem, da mulher, do idoso, dentre outras, ratificando legalmente a atribuição constitucional do governo em prover condições de acesso à saúde e de manutenção da mesma. O Ministério da Saúde é o órgão responsável pela publicação de boa parte dos documentos que normatizam as ações em saúde no âmbito do SUS além de dispor outras providencias, conforme a necessidade epidemiológica da população (WHO, 1946; Souza e Silva, Schraiber, Mota, 2019) 
As instituições de pesquisa em saúde, bem como as universidades são cruciais neste processo tendo em vista a seguridade e confiabilidade das intervenções ora propostas pelas mesmas que variam desde a elaboração de princípios, diretrizes, objetivos da mesma até manuais operacionais, notas técnicas embasadas em pesquisas científicas nacionais e mundiais, com o máximo de fidedignidade aceita, pois, parte-se do princípio que tais estudos no Brasil passam pela análise crítica e minuciosa de experts nas respectivas áreas. Fator este que também se refere à Responsabilidade Social no contexto da saúde.

\subsection{O princípio, o Estado, a pandemia e a Bioética}

O artigo 14 da DUBDH ratifica que atingir um dos mais altos padrões de saúde é um direito fundamental e que isto deve ser feito longe de qualquer situações de exclusão ou segregação de raça, cor, religião, concepção política, orientação sexual, gênero, assim como condição socioeconômica, atrelado ao progresso da ciência e das tecnologias na perspectiva de garantir ao máximo que todas as necessidades do sujeito, que variam desde suas questões individuais até questões de meio ambiente, sustentabilidade, devam ser atendidas. Por isso serão discutidas algumas ações estratégicas do governo brasileiro frente à pandemia e comportamentos destoantes do que o próprio governo "acatou" como normatizador destas ações (UNESCO 2005; Pitombeira \& Oliveira, 2020).

Em 07 de janeiro de 2020 o Ministério da Saúde, por meio da Secretaria de Vigilância Sanitária elaborou o primeiro relatório interno para gestores sobre o novo coronavírus e em fevereiro do mesmo ano foi publicado o Plano de Contingência Nacional para a Infecção Humana pelo novo coronavírus COVID-19 pelo Centro de Operações de Emergências em Saúde Pública | COE-COVID-19, também vinculado ao Ministério da Saúde e demais secretarias e órgãos de saúde envolvidos. No dia 03 de fevereiro de 2020 o Brasil decretou Emergência de Saúde Pública de Importância Nacional (ESPIN) prévia à confirmação do primeiro caso que ocorreu no mesmo mês deste decreto dando início às tomadas de decisões de controle de uma possível dizimação da doença que, infelizmente aconteceu e ainda permanece vigente (Brasil, 2020a; Brasil, 2020b; Netto $\&$ Correa, 2020).

O Plano de Contingencia Nacional foi direcionado para as Secretarias Municipais e Estaduais de Saúde, para o Governo Federal, serviços de saúde pública e privada e para demais serviços relacionados e dispõe sobre os três níveis de resposta (alerta, perigo iminente e emergência em saúde pública) para a tomada de decisão em tempo hábil pelo Ministério da Saúde caso ocorresse a circunstância emergencial de surto da infecção pelo novo coronavírus a fim de que as ações fossem coordenadas no âmbito do SUS. Para cada nível de resposta as três esferas de governo têm suas interinas responsabilidades, com vistas a manutenção da dignidade humana e segurança da população, o que pode ser exemplificado pelo decreto de Emergência de Saúde Pública de Importância Nacional (ESPIN), efetuada pelo Poder Executivo Federal a partir de ato do Ministro de Estado da Saúde, da época (Brasil, 2020b; Oliveira et al., 2020).

O primeiro caso de infecção pelo COVID-19 no Brasil surgiu na cidade de São Paulo, em fevereiro de 2020 e por conseguinte demais casos foram registrados em outras regiões e estados do Brasil sendo liberados 1 bilhão de reais aos municípios e estados para o combate da propagação do vírus e, mesmo assim, a situação se tornou ainda mais alarmante tendo em vista a rapidez da propagação do vírus, incertezas clínicas sobre mesmo, de terapêutica e possíveis instabilidades no sistema único de saúde, diante das taxas de morbidade (Aquino, 2020; Walker et al., 2020).

A Portaria no 356 de 11 de março de 2020 do Ministério da Saúde regulamenta as medidas de isolamento social e quarentena e suas especificidades, inclusive a prorrogação dos mesmos, se necessário, e atribuiu tais medidas à responsabilização dos governos municipais, estaduais e federais ou superiores em cada nível de gestão. A partir daí foram surgindo novos documentos, normativas, protocolos de manejo de todos os fatores circundantes dos processos de prevenção, controle, vigilância, tratamento relacionados ao coronavírus (Brasil, 2020c). Em 12 de março houve a confirmação do primeiro 
óbito pela doença no Brasil, uma mulher de 57 anos residente da capital paulista e atualmente as estatísticas ultrapassam o catastrófico, impactante e doloroso número de 607.462 mil óbitos causados pela COVID-19 no Brasil e 21.793 .401 casos confirmados, bem como 20.986.901 casos recuperados (Verdélio, 2020; Brasil, 2021a).

No entanto, por mais que estivessem normatizadas as medidas a serem seguidas por todos, desde a gestão, passando pelos profissionais da saúde, chegando até os usuários, pacientes e suas famílias, à população brasileira em geral, falas e atitudes de alguns membros do governo demonstraram-se opostas a tudo o que estava sendo incansavelmente estudado por grandes centros de pesquisa do mundo, normatizado pela Organização Mundial de Saúde e promulgado nos próprios documentos oriundos de autorização governamental. A comparação da doença causada pela infecção pelo novo coronavírus, à uma gripe, em diminutivo, foi um dos primeiros sinais de negacionismo de por parte do Estado, o que levou, inclusive a adesão uns e manifesto contrários de outros, no Brasil e no mundo caracterizando o governo como descrente na gravidade da situação sanitária que se instalava no país além de minimizar as medidas ora sugeridas pela Organização Mundial de Saúde, afim de conter a pandemia (Do Bu et al., 2020); Giovanella et al., 2020).

Tal achado se contrapõe ao princípio 14 da DUBH, uma vez que, houve insignificação da Responsabilidade Social do Estado no contexto da saúde o que torna a violabilidade deste princípio como real, mesmo que muitas outras ações distantes de violação da Responsabilidade Social e saúde ainda sejam executadas, e pela grande maioria dos profissionais da saúde envolvidos diretamente com a linha de frente do cuidado aos pacientes acometidos pela infecção do novo coronavírus, que também tornaram-se estatísticas de morbi-mortalidade alarmantes.

Ao se abordar a vacinação contra o novo coronavírus, mais reações governamentais com desdenha destacaram-se, quando ouviu-se que ao receber o imunobiológico a pessoa poderia se transformar em um réptil, reduzindo assim a capacidade científica dos grandes centros de pesquisa envolvidos na produção destas vacinas, além de o exercício de uma autocracia de saber deliberada por um mecanismo de poder ora político.

Cabe ressaltar que em dezembro de 2020 foi apresentada ao presidente da república a primeira edição do Plano Nacional de Operacionalização da Vacinação contra a COVID-19 que priorizou os profissionais de saúde e idosos a partir dos 75 anos de idade para serem os primeiros a receberam as duas doses da vacina, tendo em vista a exposição e maior risco de contaminação e adoecimento destes grupos. Posteriormente foram se modificando os grupos e as idades com cada município e estado aplicando a sua forma de organização do processo de vacinação, com vistas a o que foi preconizado a nível federal (Brasil, 2020d).

No vislumbre bioético, partindo-se do modelo hegemônico, ou seja, do Principialismo de Beachump e Childress, temse a não-maleficência, beneficência, autonomia e justiça a partir dos documentos e políticas ora estabelecidas, em que percebeu-se a real tentativa de incentivo e aplicação sobretudo à minimização de danos (isolamento social, quarentena, uso de máscaras, higiene das mãos, dentre outros) e maximização de benefícios (vacinação) (Beauchamp \& Childress, 1979).

No que se refere ao princípio da justiça, um dos grandes desafios da inequânime realidade deste país e que teve seu destaque na priorização dos profissionais da saúde da linha de frente do combate ao novo coronavírus como grupo prioritário e primário para vacinação. Muitos destes profissionais tornaram-se incapacitados pelo coronavírus e inicialmente uma proposta de indenização a estes havia sido negada pelo governo federal, porém o veto foi derrubado pelo congresso e a lei tornou-se promulgada. Tal referência traz à tona a proposta do médico, cientista e político italiano Giovanni Berlinguer (1988) que dispôs em seu discurso que a saúde pode ser transformada a partir de dadas providencias e uma delas é a vida profissional saudável. Berlinguer que o direito à saúde e a uma vida plena são condições essenciais para a existência da ética e que está é característica fiel da democracia, corroborando assim com a proposta do artigo 14 da DUBDH e não com atitudes ditatoriais (Brasil, 2021b; Santos \& Cornelli, 2019). 
A Bioética de Intervenção (BI) enquanto referencial latinoamericano, se preocupa com as ações cotidianas relacionadas à saúde, porém, aplicada à realidade de países periféricos assim como o Brasil, que possuem nas vulnerabilidades e na exclusão social fatores que mimetizam a possibilidade de acesso à saúde, bem como a demais direitos. Atender as necessidades de cada pessoa conforme as peculiaridades destas, considerando também o pluralismo moral são alguns dos pilares que norteiam a Bioética de Intervenção no tocante ao acesso à saúde e cabe ao Estado a atenção e priorização dos indivíduos mais vulneráveis, ou seja, para além da Responsabilidade Social inerente ao Estado tem-se a especificidade e ajustar isto aos que mais precisam (Garrafa, 2005; Nascimento \& Martorell, 2013).

Numa situação pandêmica como a atual, todos tornam-se vulneráveis em diversas escalas, por isso a tomada de decisão governamental deve ser ágil, segura e bem estratégica tendo em vista o forte impacto que o não planejamento destas ações podem acarretar, como bem tem sido exemplificado pelo protelamento da compra ou produção de vacinas para o povo brasileiro, pelo má exemplo de alguns governantes em relação aos cuidados para a prevenção da infecção e disseminação do novo coronavírus como a persistência em não utilizar máscara em eventos que grande número de pessoas e independente disto, além de promoção deliberadas de aglomerações e de eventos que insignificam a atual estatística de mais de 420 mil pessoas mortas pelo COVID, no Brasil.

\section{Considerações Finais}

A Responsabilidade Social e a Saúde enquanto princípio de grande responsabilidade estatal para o seu cumprimento, foi visivelmente violado na grande maioria das ações governamentais exercidas até o presente momento, contrariando algumas das próprias decisões autorizadas pelo governo, fator este que traz à tona uma certa instabilidade no potencial de gestão que se esperava mais eficaz, sobretudo, em um grande momento de saúde pública como tem sido a pandemia no novo coranavírus (SARS-COV-2).

O pensamento bioético, com ênfase na Bioética de Intervenção (BI) deveria ser premissa para alguns governantes, uma vez que, dela partem todas as demais "identidades" da população brasileira sobretudo no quesito inequanimidade, possivelmente proveitosa ao exercício governamental neste país. Enseja-se mais pesquisas sobre o artigo 14 da Declaração Universal sobre Bioética e Direitos Humanos, bem como à luz de outros princípios como - Respeito pela Vulnerabilidade Humana e pela Integridade Individual; Igualdade, Justiça e Equidade e Benefício e dano, que também podem ser aplicáveis ao contexto pandêmico a fim de que as reais intenções no âmbito de preservar a dignidade humana e os direitos essenciais das pessoas sejam reais e concretas.

\section{Referências}

Aquino V. (2020). Ministério de Saúde. Brasil registra 2.915 casos confirmados de coronavírus e 77 mortes. https://www.saude.gov.br/noticias/ag enciasaude/46610-brasil-registra-2-915-casosconfirmados-de-coronavirus-e-77-mortes .

Beauchamp, T., \& Childress, J. (1979). Principles of biomedical ethics. Oxford University Press. 314 p.

Berlinguer G. (1988). A doença. Hucitec, p. 15.

Borde, E., Hernández-Álvarez, M., \& Porto, M. F. S. (2015). Uma análise crítica da abordagem dos Determinantes Sociais da Saúde a partir da medicina social e saúde coletiva latino-americana. Saúde Debate, 39 (106), 841-54. 10.1590/0103-1104201510600030023.

Brasil. (1988). Constituição da República Federativa do Brasil. Senado Federal;

Brasil (2021a). Coronavírus/Brasil. Painel Coronavírus. Atualizado em 29/10/2021. Óbitos confirmados. https://covid.saude.gov.br/. Acesso em 30 out. 2021.

Brasil. (2021b). Lei $\mathrm{n}^{\circ} 14.128$ de 26 de março de 2021. Dispõe sobre compensação financeira a ser paga pela União aos profissionais e trabalhadores de saúde que, durante o período de emergência de saúde pública de importância nacional decorrente da disseminação do novo coronavírus (SARS-CoV-2). Diário Oficial da União. Brasília, seção 1, pág. 4, 26 de março.

Brasil. (1990). Lei $\mathrm{n}^{\circ}$ 8080, de 19 de setembro de 1990. Dispõe sobre as condições para a promoção, proteção e recuperação da saúde, a organização e o funcionamento dos serviços correspondentes e dá outras providências. Diário Oficial da União. Brasília, p. 18055, col. 1, 20 set. 
Brasil. (2020a). Ministério da Saúde, Centro de Operações em Emergências em Saúde Pública (COE-COVID-19). Plano de Contingência Nacional para a Infecção Humana pelo novo coronavírus COVID-19. Brasília: Ministério da Saúde; Disponível: https://portalarquivos2.saude.gov.br/images/pdf/2020/fevereiro/13/plano-contingencia-coronavirus-COVID19.pdf

Brasil. (2020b). Ministério da Saúde, Plano Nacional de Operacionalizaçao da vacinação contra a COVID-19. Ministério da Saúde; Disponível: https://www.gov.br/saude/pt-br/media/pdf/2020/dezembro/16/plano_vacinacao_versao_eletronica.pdf

Brasil. (2020c). Ministério da Saúde. Portaria MS/GM n. 188, de 3 de fevereiro de 2020. Declara Emergência em Saúde Pública de importância Nacional em decorrência da Infecção Humana pelo novo Coronavírus (2019-nCoV). Diário Oficial da União, Brasília. http://www.planalto.gov.br/ccivil_03/portaria/prt188-20-ms.htm

Brasil. (2020d). Ministério da Saúde. Portaria $\mathrm{n}^{\circ} 356$ de 11 de março de 2020. Dispõe sobre a regulamentação e operacionalização do disposto na Lei $n^{o}$ 13.979, de 6 de fevereiro de 2020, que estabelece as medidas para enfrentamento da emergência de saúde pública de importância internacional decorrente do coronavírus (COVID-19). Brasília: Ministério da Saúde. https://www.in.gov.br/en/web/dou/-/portaria-n-356-de-11-de-marco-de-2020-247538346

Carrapato, P, Correia, P, \& Garcia, B. (2017). Determinante da saúde no Brasil: a procura da equidade na saúde. Saúde Soc. 26(3): 676-689. 10.1590/S010412902017170304 .

Do Bú, E. A., Alexandr,e M. E. S., Bezerra V. A. S., Sá-Serafin R. C. N., \& Coutinho M. P. L. (2020) Representações e ancoragens sociais do novo coronavírus e do tratamento da COVID-19 por brasileiros. Estudos de Psicologia, 37(e200073). 10.1590/1982-0275202037e200073.

Duarte E., Eble L. J, \& Garcia L. P. (2018). 30 anos do Sistema Único de Saúde. Epidemiol. Serv. Saúde, 27 (1). 10.5123/s1679-49742018000100018.

Feitosa S. F., \& Nascimento W. F. (2015). A bioética de intervenção no contexto do pensamento latino-americano contemporâneo. Rev. bioét. 23(2), 277-84. $10.1590 / 1983-80422015232066$.

Garrafa V, \& Porto D. (2003). Intervention bioethics: a proposal for peripheral countries in a context of power and Injustice. Bioethics, 17 (5-6), 399416. $10.1111 / 1467-8519.00356$

Garrafa V. (2005). Da bioética de princípios à uma bioética interventiva. Bioética, 13 (1). https://revistabioeti ca.cfm.org.br/index.php/revista_bioetica/article/view/97/102.

Giovanella L, Medina M. G, Aquino R, \& Bousquat A. (2020). Negacionismo, desdém e mortes: notas sobre a atuação criminosa do governo federal brasileiro no enfrentamento da COVID-19. Saúde Debate, 44(126), 895-901. 10.1590/0103-1104202012623.

Mattos R. A. (2009). Princípios do Sistema Único de Saúde (SUS) e a humanização das práticas de saúde. Debate Interface, 13 (suppl 1), 771-80. 10.1590/S141432832009000500028 .

Nascimento, W. F.; \& Martorell, L. B. (2013). A bioética de intervenção em contextos descoloniais. Rev. bioét. (Impr.). Brasília-DF, $21(3), 423-31$. https://www.scielo.br/j/bioet/a/DB3X3vCy3TwKLbVFwJ9fhnk/?lang=pt.

Netto R. G. F, \& Correa J. W. N. (2020). Epidemiologia do surto de doença por coronavírus (COVID-19). Revista Desafios. 7(Supl. COVID-19). 10.20873/uftsuple2020-8710.

Oliveira W. K. et al. (2020). Como o Brasil pode deter a COVID-19. Epidemiol. Serv. Saúde, Brasília, 29(2), 1-8. DOI: 10.5123/S1679-49742020000200023.

Organização das Nações Unidas para a Educação, a Ciência e a Cultura (Unesco). (2005). Declaração Universal sobre Bioética e Direitos Humanos. Paris: Unesco; http://bvsms.saude.gov.br/bvs/publicacoes/declaracao_univ_bioetica_dir_hum.pdf

Paim J. S. (2019). Os sistemas universais de saúde e o futuro do Sistema Único de Saúde (SUS). Saúde debate, 43(spe5). 10.1590/0103-11042019S502 .

Pitombeira D. F., \& Oliveira 1. C. (2020). Pobreza e desigualdades sociais: tensões entre direitos, austeridade e suas implicações na atenção primária. Ciênc. saúde coletiva. 25(5), 1699-1708. 10.1590/1413-81232020255.33972019.

Santos SG, Cornelli G. (2019). Ontologia política da doença: em defesa da saúde pública. Rev. Bioét, 27(2), 204-11. 10.1590/1983-80422019272302.

Schaefer, B. M., Resende, R. C., Epitácio, S. S. F., \& Aleixo, M. T. (2020). Ações governamentais contra o novo coronavírus: evidências dos estados brasileiros. Revista de Administração Pública, 54(5), 1429-1445. DOI: 10.1590/0034-761220200503.

Silva, M. S., \& Gomes Filho, A. S. (2020). Responsabilidade Social empresarial: uma revisão de literatura (2018-2019). Entrepreneurship, 4(2), 37-42. 10.6008/CBPC2595-4318.2020.002.0004.

Souza e Silva M. J., Schraiber L. B., \& Mota A. (2019). O conceito de saúde na Saúde Coletiva: contribuições a partir da crítica social e histórica da produção científica. Physis: Revista de Saúde Coletiva, 29(1), e290102, 1-18. https://www.e-publicacoes.uerj.br/index.php/physis/article/view/43079 .

Verdélio A (2020). Primeira morte por Covid-19 no Brasil aconteceu em 12 de março. Agência Brasil. <https://agenciabrasil.ebc.com.br/saude/noticia/202006/primeira-morte-por-covid-19-no-brasil-aconteceu-em-12-de-marco>.

Walker P. G. T., Whittaker C., Watson O., Baguelin M., Ainslie K. E. C., Sangeeta, B., et al. (2020). The Global Impact of COVID-19 and Strategies for Mitigation and Suppression. Imperial College London. https://doi.org/10.1126/science.abc0035 .

World Health Organization (WHO). (1946). WHO remains firmly committed to the principles set out in the preamble to the Constitution. Geneva: WHO. https://www.who.int/about/governance/constitution .

World Health Organization. WHO (2020). Director-General's opening remarks at the media briefing on COVID-19. Geneva: WHO. https://www.who.int/director-general/speeches/detail/who-director-general-s-opening-remarks-at-the-media-briefing-on-covid-19---11-march-2020 . 